\title{
Effects of Central Tube on Shape of Modal Duct of a Helicoid in a Simple Expansion Chamber
}

\author{
Daniel Omondi Onyango $\mathbb{C}^{1},{ }^{1}$ Robert Kinyua, ${ }^{1}$ and Abel Nyakundi Mayaka ${ }^{2}$ \\ ${ }^{1}$ Jomo Kenyatta University of Agriculture and Technology, Kenya \\ ${ }^{2}$ Multimedia University of Kenya, Kenya \\ Correspondence should be addressed to Daniel Omondi Onyango; domondi@eng.jkuat.ac.ke
}

Received 9 April 2018; Accepted 2 December 2018; Published 11 December 2018

Academic Editor: Benjamin Soenarko

Copyright (c) 2018 Daniel Omondi Onyango et al. This is an open access article distributed under the Creative Commons Attribution License, which permits unrestricted use, distribution, and reproduction in any medium, provided the original work is properly cited.

\begin{abstract}
The shape of the modal duct of an acoustic wave propagating in a muffling system varies with the internal geometry. This shape can be either as a result of plane wave propagation or three-dimensional wave propagation. These shapes depict the distribution of acoustic pressure that may be used in the design or modification of mufflers to create resonance at cut-off frequencies and hence achieve noise attenuation or special effects on the output of the noise. This research compares the shapes of acoustic duct modes of two sets of four pitch configurations of a helicoid in a simple expansion chamber with and without a central tube. Models are generated using Autodesk Inventor modeling software and imported into ANSYS 18.2, where a fluid volume from the complex computer-aided-design (CAD) geometry is extracted for three-dimensional (3D) analysis. Mesh is generated to capture the details of the fluid cavity for frequency range between 0 and $2000 \mathrm{~Hz}$. After defining acoustic properties, acoustic boundary conditions and loads were defined at inlet and outlet ports before computation. Postprocessed acoustic results of the modal shapes and transmission loss (TL) characteristics of the two configurations were obtained and compared for geometries of the same helical pitch. It was established that whereas plane wave propagation in a simple expansion chamber (SEC) resulted in a clearly defined acoustic pressure pattern across the propagation path, the distribution in the configurations with and without the central tube depicted three-dimensional acoustic wave propagation characteristics, with patterns scattering or consolidating to regions of either very low or very high acoustic pressure differentials. A difference of about 80 decibels between the highest and lowest acoustic pressure levels was observed for the modal duct of the geometry with four turns and with a central tube. On the other hand, the shape of the TL curve shifts from a sinusoidal-shaped profile with well-defined peaks and valleys in definite multiples of $\pi$ for the simple expansion chamber, while that of the other two configurations depended on the variation in wavelength that affects the location of occurrence of cut-on or cut-off frequency. The geometry with four turns and a central tube had a maximum value of TL of about 90 decibels at approximately $1900 \mathrm{~Hz}$.
\end{abstract}

\section{Introduction}

In vortex motion, the curvature of the streamlines introduces the action of centrifugal force which must be counterbalanced by a pressure gradient in the fluid [1]. Aerodynamic sound is generated as a result of the movement of vortices, or of vorticity, in an unsteady fluid flow. Vortex flow can be introduced in fluid systems by modification of the path of flow in order to achieve desired effects. Such modifications include introduction of holes with sharp edges [2], barriers in the path of flow [3], or sudden contraction or expansion as is the case with the SEC [4]. Applications of this phenomenon in vortex motion have been successfully studied by Smith et al., [5] who identified two scattering mechanisms that allow neighbouring modes to interact; scattering occurs at significantly lower frequencies when the mean flow is present; an exchange of energy between mean flow and acoustic field occurs during scattering.

Effects of vortex in acoustics have also been studied by Oosterhuis et al., [6] who predicted the relationship between pressure drop and acoustic power dissipation. Changes in circulation or area of a vortex ring gives rise to a dipole sound field. When this sound source couples with a resonator, large amplitudes may be generated. The nonlinear behaviour of 
resonators where change of the output is not proportional to the change of the input occurring with flow separation makes such devices efficient sound absorbers. An acoustic wave may be greatly affected by factors such as mean flow, convection, refraction in shear, coupling with vorticity, and scattering by turbulence.

The configuration of the helicoid and the central tube in a simple expansion chamber which is the subject of this investigation is a modified version of the Herschel-Quincke tube [7]. In the present arrangement, the path bypassing the central tube is made longer by varying the pitch of the helicoid for a fixed length of the chamber. Similar modifications have been studied by Selamet et al. [8], Karlsson and Glav [9], and Selamet and Easwaran [10], though the difference here is the elongation of the path along and around the axis of propagation as opposed to that parallel to the axis of propagation only in the Herschel-Quincke version.

The wave equation describing sound in one dimension at a position $\mathrm{x}$ is given by [11]

$$
\frac{\partial^{2} p}{\partial x^{2}}-\frac{1}{c^{2}} \frac{\partial^{2} p}{\partial t^{2}}=0
$$

where $\boldsymbol{p}$ is the acoustic pressure and $\boldsymbol{c}$ is the speed of sound. Provided that the speed is a constant and not dependent on frequency, then the general solution of the equation becomes

$$
p=f(c t-x)+(g(c t+x)
$$

where $f$ and $g$ are any two twice differentiable functions. This may be pictured as the superposition of two waveforms of arbitrary profiles, one $(f)$ travelling up the $\mathrm{x}$-axis and the other $(\boldsymbol{g})$ down the $\mathrm{x}$-axis at speed $\boldsymbol{c}$. The particular case of a sinusoidal wave traveling in one direction is obtained by choosing either $\boldsymbol{f}$ or $\boldsymbol{g}$ to be a sinusoid and the other to be zero, giving

$$
p=p_{o} \sin \omega t \pm k x
$$

where $\boldsymbol{w}$ is the angular frequency of the wave and $\boldsymbol{k}$ is its wave number.

\section{Materials and Methods}

Two arrangements (Model A and Model B) each with four variations of the helicoid within a simple expansion chamber of overall dimensions as indicated in Figure 1(a) were used in this analysis. Model A had been investigated by [12]. The helicoid geometry (Figure 1(b)) is defined by its outer diameter which coincides with the inner diameter of the expansion chamber $(\mathrm{D}=150 \mathrm{~mm})$, inner diameter corresponding to the inner diameter of the inlet and outlet pipes $(\mathrm{d}=50 \mathrm{~mm})$, pitch that varies $(350,175,117$, and $87.5 \mathrm{~mm})$, and an overall length corresponding to the inner length of the expansion chamber $(\mathrm{L}=350 \mathrm{~mm})$. The thickness of the model is $5 \mathrm{~mm}$ throughout.

For the first arrangement, acoustic propagation is such that flow is possible through the mid-section and around the spaces after sudden expansion at the entry to the chamber defined by the helicoid within the SEC and later joining the main stream due to contraction at the end of the expansion chamber. Effects of back-pressure are minimized since there is no barrier in the direct path of propagation of the acoustic wave, but possibility of generation of aerodynamic sound exists as a result of vorticity created by the helicoid. In the second arrangement, a concentric tube of $50 \mathrm{~mm}$ diameter is added at the expansion chamber for all the variations shown in the second column in Table 1 . A space of $50 \mathrm{~mm}$ is created by the concentric tube immediately after the sudden expansion and just before the sudden contraction of the SEC.

The geometries were modeled in 3D using Autodesk Inventor software and imported into Ansys, where fluid volume from the complex CAD geometry was extracted for 3D analysis. The geometry was meshed based on the maximum frequency of $2000 \mathrm{~Hz}$ and sonic speed of 343 $\mathrm{m} / \mathrm{s}$, giving a mesh size of approximately $0.02 \mathrm{~m}$. Transient Computational Fluid Dynamics (CFD) analysis was carried out after definition of acoustic properties and application of acoustic boundary conditions and loads. Details of boundary conditions defined are included in the Appendix at the end of this paper.

\section{Results and Discussions}

Postprocessed results included a map of acoustic sound pressure field computed at each element node, acoustic time frequency plot, and acoustic power result plot. These are summarized in Figures 2-5 and in Table 2.

In Figure 2, the acoustic pressure map shows a clearly defined pattern characteristic of plane waves propagation defined by (3). It represents a time-harmonic sound field of similar characteristics in three distinct sections (the inlet section, the outlet section, and the chamber section) that are independent of the axial coordinate defined by an infinite sum of special solutions called nodes that retain their shape when travelling through each of these sections. An overall TL of about 15 decibels is exhibited and is evident from the colour scheme as read from the legend on the left.

From the geometry of the basic structure, the area expansion ratio $(\mathrm{m}=\mathrm{D} / \mathrm{d})$ is 3 while the ratio $\mathrm{L} / \mathrm{D}=7$ implying that the chamber used is axially longer. The speed of sound $c$ is taken as $343.14 \mathrm{~ms}^{-1}$. In Figure 3, TL of the SEC is presented. Its axial TL is given by [11]

$$
T L=10 \log \left\{1+\left(\frac{m-1 / m}{2} \sin k l\right)^{2}\right\}
$$

From (4), for all values of $m$, TL would tend to zero at the Helmholtz number (or nondimensional frequency parameter) $k l$, given by

$$
k l=m \pi \longrightarrow \frac{2 \pi f}{c} l=m, \quad m=0,1,2,3, \ldots
$$

The frequency of the $m^{\text {th }}$ trough $f_{m}$ is given by

$$
f_{m}=\frac{m c}{2 l} H z \text {. }
$$

It shows peaks and troughs in a uniform pattern, with troughs occurring periodically at approximately 500 hertz $(\mathrm{Hz})$ and 


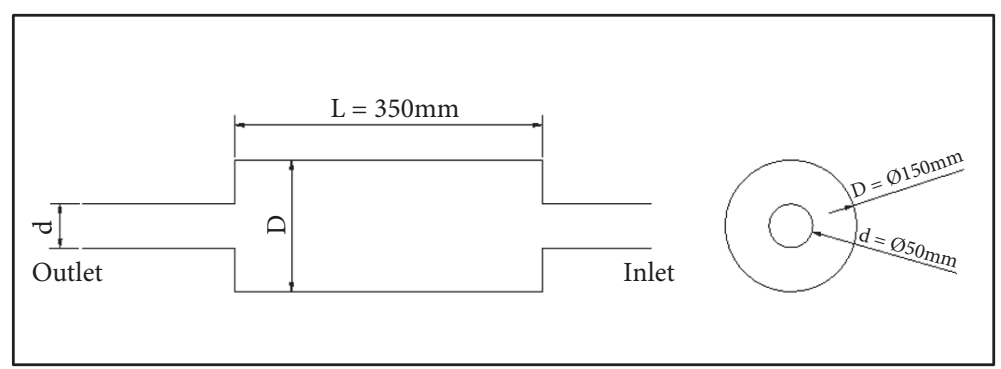

(a) Overall dimensions and orientation of the SEC

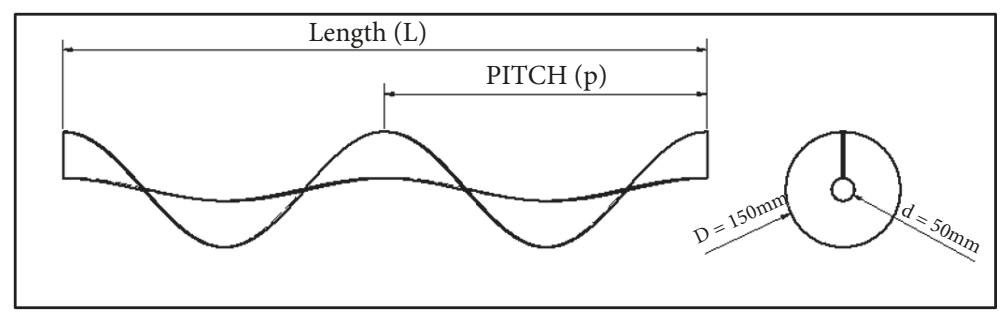

(b) Nomenclature of the Helicoid geometry

Figure 1

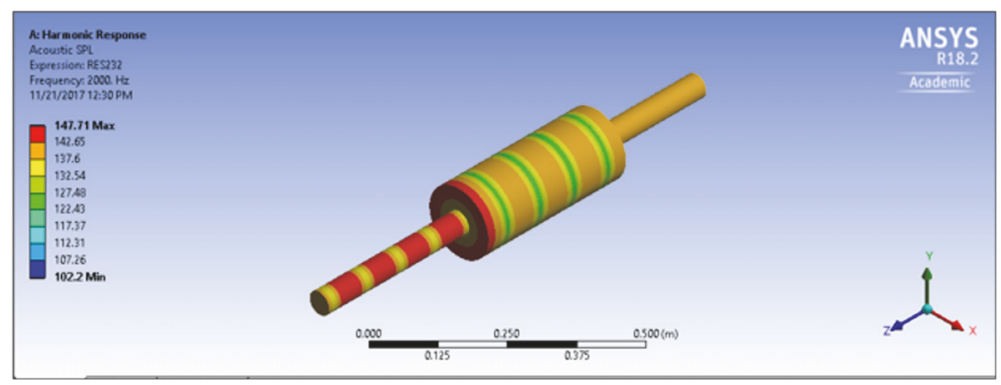

Figure 2: Acoustic pressure map for the Simple Expansion Chamber.

TABLE 1: Configurations of test specimens with and without a central tube.

S.NO. MODEL A - WITHOUT TUBE


Table 2: Acoustic Pressure maps for models with and without central tube.

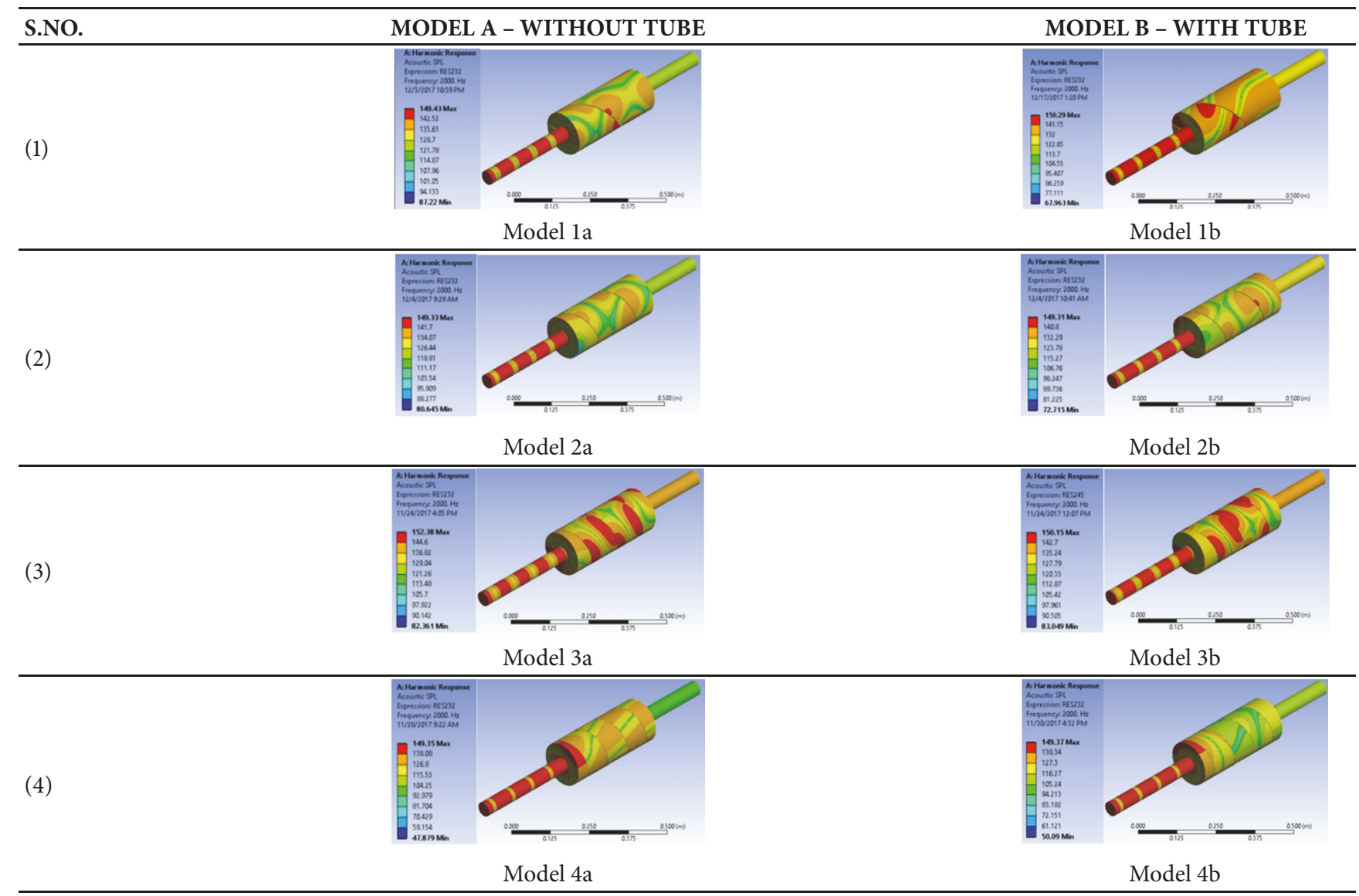

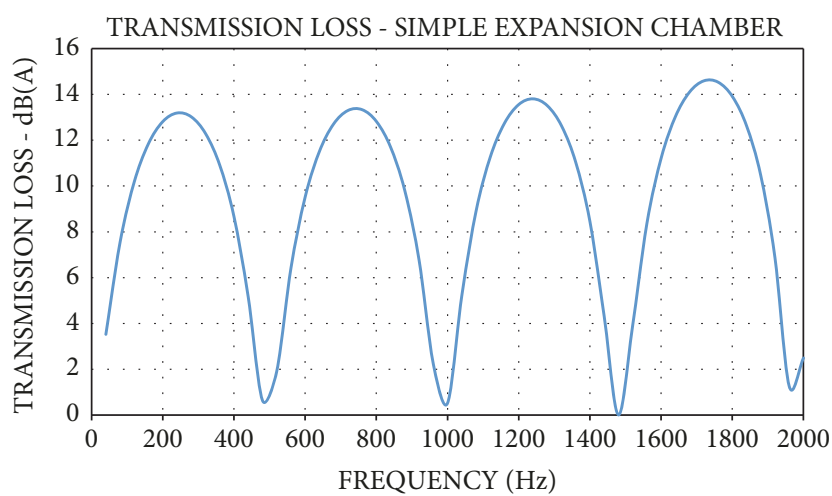

Figure 3: Transmission Loss of a Simple Expansion Chamber.

its integral multiples. At these locations, the TL is or tends to zero, implying that all the acoustic energy radiates through the outlet of the geometry. Also, from the graph, the average TL (at the peaks) is similar to that obtained from the modal duct map and confirms the findings for the whole range of frequencies under investigation. This can also be seen from (4) which indicates that the peaks would occur at $k l=(2 \mathrm{~m}$ - 1) $\pi / 2$, where $m=1,2,3, \ldots$; that is midway between the adjacent troughs. Most of the acoustic energy injected is reflected by the cavity and comes back through the inlet

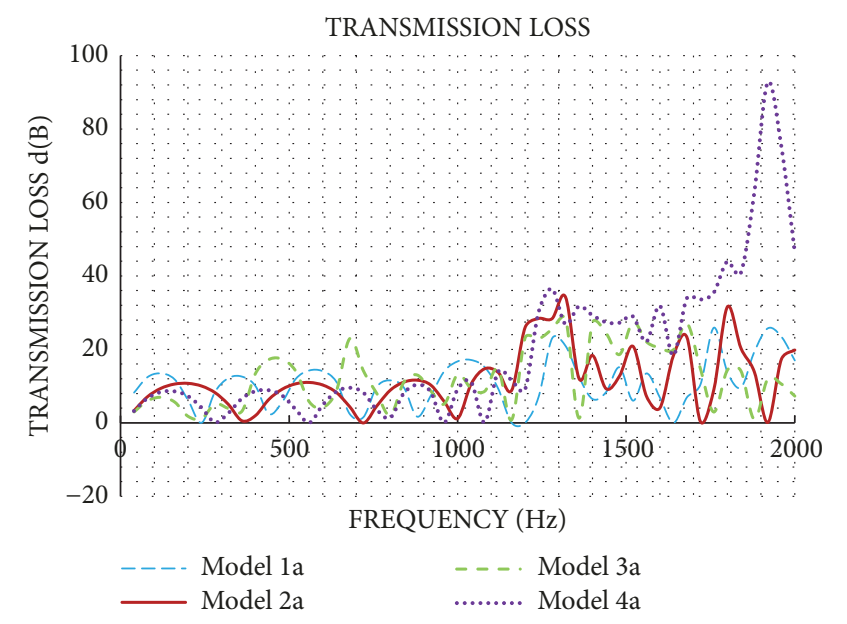

FIGURE 4: Transmission loss of helicoid without central tube.

section. This result (Figures 2 and 3 ) is similar to that obtained by several researchers $[13,14]$.

Equation (4) also indicates that an increase in area ratio increases TL in all frequencies but does not raise troughs; they still touch $0 \mathrm{~dB}$. This makes it not suitable for normal use as achieving a desired TL characteristic would mean increase in muffler size that is undesirable especially since the 


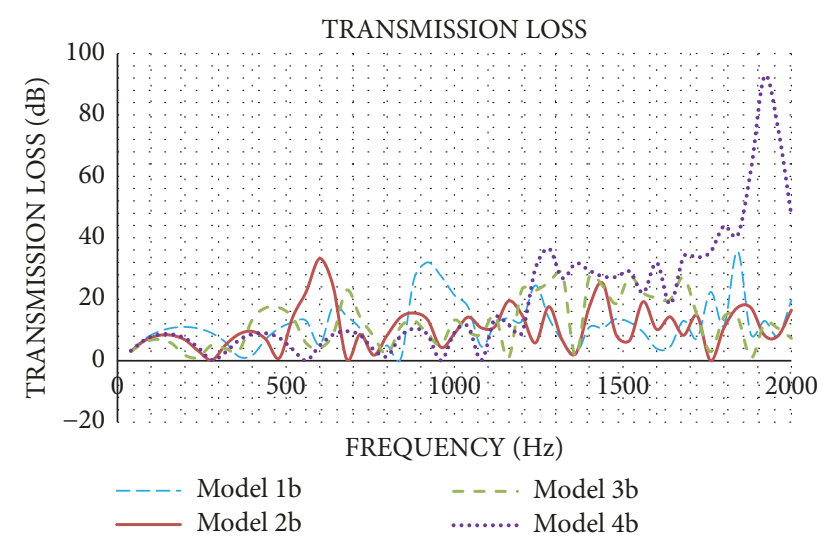

FIgURE 5: Transmission Loss of helicoid with central tube.

undercarriage is normally limited in space. The increase in size would also translate to increase in costs.

The results of the maps in Table 2 show plots of the real part of the acoustic pressure in order to visualize resonance observed in the TL plot for each of the models evaluated (Model a column 1 and Model b column 2). These are distinctly different in configuration from those observed for the SEC. The helicoid introduces a torsional effect on the regions of low and high acoustic pressures, thereby accounting for the different profiles of the TLs. In these models, the influence of the helicoid is caused by centrifugal effects of the vortex that results in pressure drops and acoustic power dissipation giving rise to a dipole sound field. Model la exhibits characteristics of plane wave propagation up to about $1200 \mathrm{~Hz}$ where 3D acoustic wave propagation starts. TL is a maximum at about $1300 \mathrm{~Hz}$. In comparison with Model 1b, 3D acoustic wave propagation is experienced after $400 \mathrm{~Hz}$ with maximum TL occurring at around $900 \mathrm{~Hz}$.

For Model 2a, a sudden spike in the value of TL sets in at about $1150 \mathrm{~Hz}$ while, for Model $2 \mathrm{~b}$, this occurs much early at $600 \mathrm{~Hz}$. For Model 3a, the sudden spike in the value of TL is observed at $700 \mathrm{~Hz}$ while for Model $3 \mathrm{~b}$ this occurs at about $450 \mathrm{~Hz}$. For these three sets of models, effects of 3D wave propagation are experienced much earlier with the central tube in place as compared with when the tube is not included. Both Models 4a and 4b exhibit the same characteristics where effects of 3D wave propagation set in about $1400 \mathrm{~Hz}$ with a maximum TL of 92 decibels occurring at $1900 \mathrm{~Hz}$. A broadband attenuation is observed for Model 4 for frequency range between $1400 \mathrm{~Hz}$ and $1900 \mathrm{~Hz}$. However, the similarities in TL characteristics do not reflect in the acoustic pressure maps, but the geometries with and without the central tube appear to produce the same TL characteristics.

With the introduction of the helicoid within the SEC, regions of fairly low acoustic pressure are observed throughout this section. The occurrence of these low-pressure regions is likely to affect the overall acoustic pressure in the respective configurations. As the wave propagates downstream, there is a general reduction in acoustic pressure levels with patterns indicating noise attenuation. As the number of turns increases from one to four, the pattern also modifies to give an overall low acoustic pressure downstream as seen in Model 4b in Table 2. This phenomenon translates to an improved transmission loss characteristic of the geometry. The effective parameters along the propagation direction within the helicoid are dependent on helicity as the pitch varies when other geometric parameters remain constant.

\section{Conclusions and Recommendations}

In this paper, Ansys 18.2 software has been used to simulate acoustic energy distribution in a simple expansion chamber with helicoid. Four variations of the helicoid have been used to predict the behaviours of acoustic energy as the pitch is varied. Results present the transformation of acoustic energy due to plane wave propagation as observed for a simple expansion chamber to a complex combination of plane wave and 3-dimensional wave propagation induced by the helicoidal geometry. Consolidation and reconfiguration of regions of high and low acoustic pressures are observed. The inclusion of the central tube provides two distinct paths for the propagation of acoustic waves. This leads to acoustic wave cancellation and hence noise attenuation if there is a phase difference between the two propagating waves as they meet downstream. The introduction of the central tube also contributed to the change in acoustic energy distribution.

Transmission loss characteristics of four variations of a helicoid in a simple expansion chamber have also been investigated. The TL curves show "peaks" for which the muffler is efficient and "valleys" where TL approaches or tends to zero indicating that all the acoustic energy radiates through the outlet of the muffler geometry. At the maximum TL, most of the acoustic energy injected is reflected by the cavity and comes back through the inlet section.

At lower frequencies, results of the models show plane wave propagation characterized by uniform and periodic shape of the transmission loss graph. This can also be seen in the pressure field map of respective models downstream and upstream of the expansion chamber. At higher frequencies, however, complex pressure fields are formed indicating that wave propagation is not planer anymore and thus will not favour acoustic propagation. This is indicated in the pressure field map in the simple expansion volume by the different pressure levels with the different colour shades. An interpretation of this on the transmission loss graph is the high transmission loss values that follow the plane wave propagation profile on the graphs.

From the analysis of the acoustic maps around the helicoid fitted in a simple expansion chamber, the geometry can be further developed to improve transmission loss characteristics of the geometry and hence higher noise attenuation.

\section{Appendix}

\section{Settings for Simulation Using Ansys 18.2}

(1) Analysis settings

Frequency spacing: Linear

Range (min and max): 0 to 2000

Solution interval: 50 
Solution method: Full

Output controls: General Miscellaneous

(2) Acoustic Body

Mass density: $1.204 \mathrm{kgm}^{-3}$

Sound speed: $343.24 \mathrm{~ms}^{-1}$

Acoustic-structural coupled body: Uncoupled with symmetric Algorithm

Behaviour: Compressible

(3) Other boundary conditions:

Acoustic normal surface velocity: $-1 \mathrm{~ms}^{-1}$ at inlet, and 0 phase angle

Transparent port setting specified for inlet and outlet

Acoustic radiation boundary defined for the inlet and outlet.

\section{Data Availability}

The data used to support the findings of this study are available from the corresponding author upon request.

\section{Conflicts of Interest}

The authors declare that they have no conflicts of interest.

\section{Acknowledgments}

The authors acknowledge the financial support offered by the Africa-ai-Japan Project towards this research.

\section{References}

[1] J. F. Douglas, J. M. Gasiorek, and J. A. Swaffield, Journal of Fluid Mechanics, Longman, England, 2nd edition, 1993.

[2] S. Weyna and W. Mickiewicz, "Multi-modal acoustic flow decomposition examined in a hard walled cylindrical duct," Archives of Acoustics, vol. 39, no. 2, pp. 289-296, 2014.

[3] Z. Parlar, S. Ari, R. Yilmaz, E. Ozdemir, and A. Kahraman, "Acoustic and flow field analysis of a perforated muffler design," International Journal of Mechanical, Aerospace, Industrial, Mechatronic, and Manufacturing Engineering, vol. 7, no. 3, pp. 447-451, 2013.

[4] W.-H. Tan, T. S. Khor, and N. H. Zunaidi, "Development of acoustical simulation model for muffler," International Journal of GEOMATE, vol. 11, no. 2, pp. 2385-2390, 2016.

[5] A. F. Smith, N. C. Ovenden, and R. I. Bowles, "Flow and geometry induced scattering of high frequency acoustic duct modes," Wave Motion, vol. 49, no. 1, pp. 109-124, 2012.

[6] J. P. Oosterhuis, S. Bühler, T. H. Van Der Meer, and D. Wilcox, "A numerical investigation on the vortex formation and flow separation of the oscillatory flow in jet pumps," The Journal of the Acoustical Society of America, vol. 137, no. 4, pp. 1722-1731, 2015.

[7] G. W. Stewart, "The theory of the Herschel-Quincke tube," Physical Review A: Atomic, Molecular and Optical Physics, vol. 31, no. 4, pp. 696-698, 1928.
[8] A. Selamet, N. S. Dickey, and J. M. Novak, "The HerschelQuincke Tube: A Theoretical, Computational, And Experimental Investigation," The Journal of the Acoustical Society of America, vol. 96, no. 5, pp. 3177-3185, 1994.

[9] M. Karlsson and R. Glav, "The Flow Reversal Resonator," in Proceedings of the SAE 2007 Noise and Vibration Conference and Exhibition, 2007.

[10] A. Selamet and V. Easwaran, "Modified Herschel-Quincke tube: Attenuation and resonance for n-duct configuration," The Journal of the Acoustical Society of America, vol. 102, no. 1, pp. 164-169, 1997.

[11] M. L. Munjal, Acoustics of Ducts and Mufflers, John Wiley \& Sons, Chichester, 2nd edition, 2014.

[12] D. O. Onyango, R. Kinyua, and A. N. Mayaka, "Experimental Investigation of a helicoid as an exhaust noise control element," 2017.

[13] Z. Tao and F. A. Saybert, A review of current technologies for measuring muffler Transmission Los, SAE International, Travers City, Mich, USA.

[14] K. S. Anderson, "Analyzing muffler performance using the Transfer Matrix Method," in COMSOL Conference, 2008. 


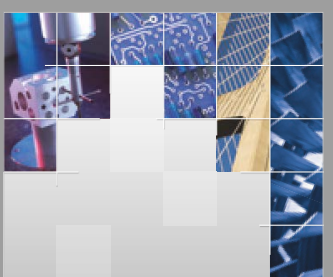

\section{Enfincering}
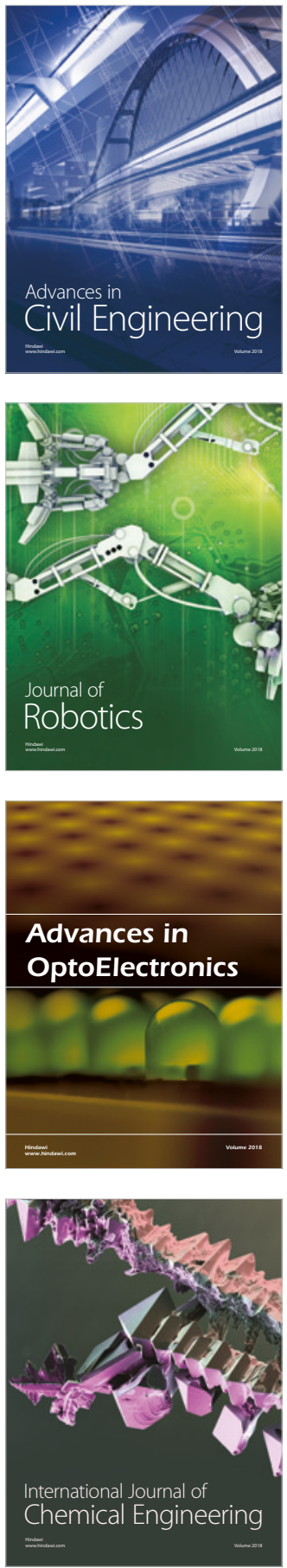

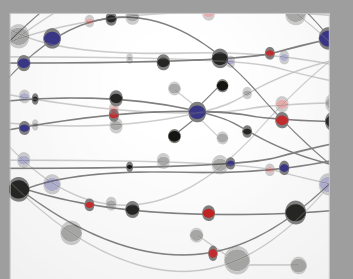

\section{Rotating \\ Machinery}

The Scientific World Journal

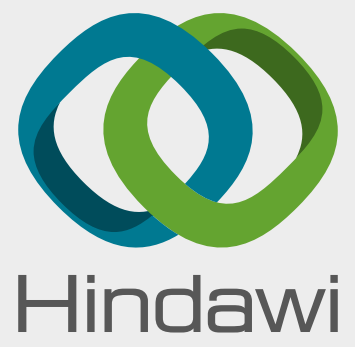

Submit your manuscripts at

www.hindawi.com
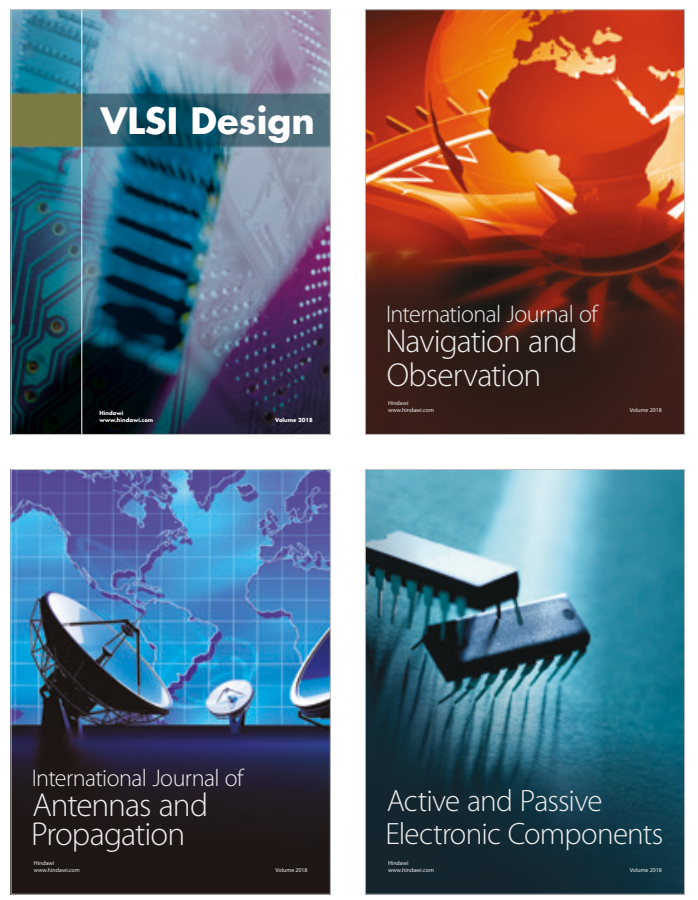
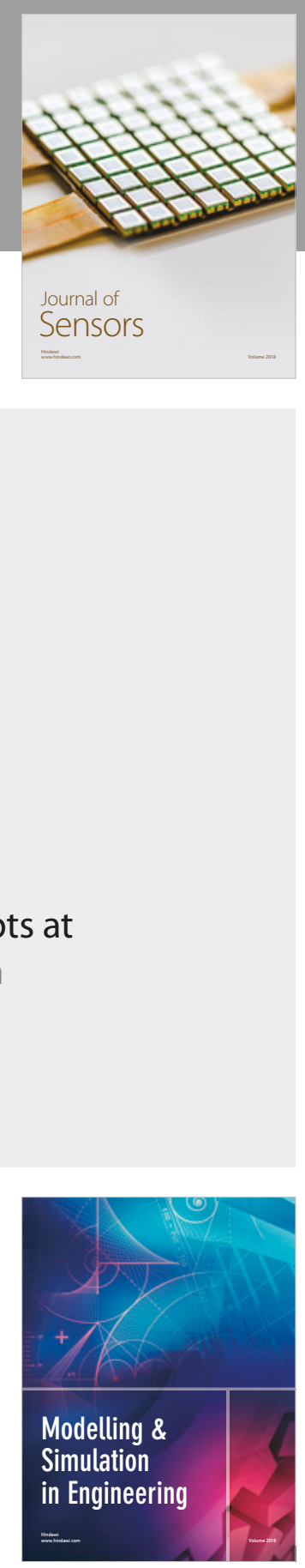

\section{Advances \\ Multimedia}
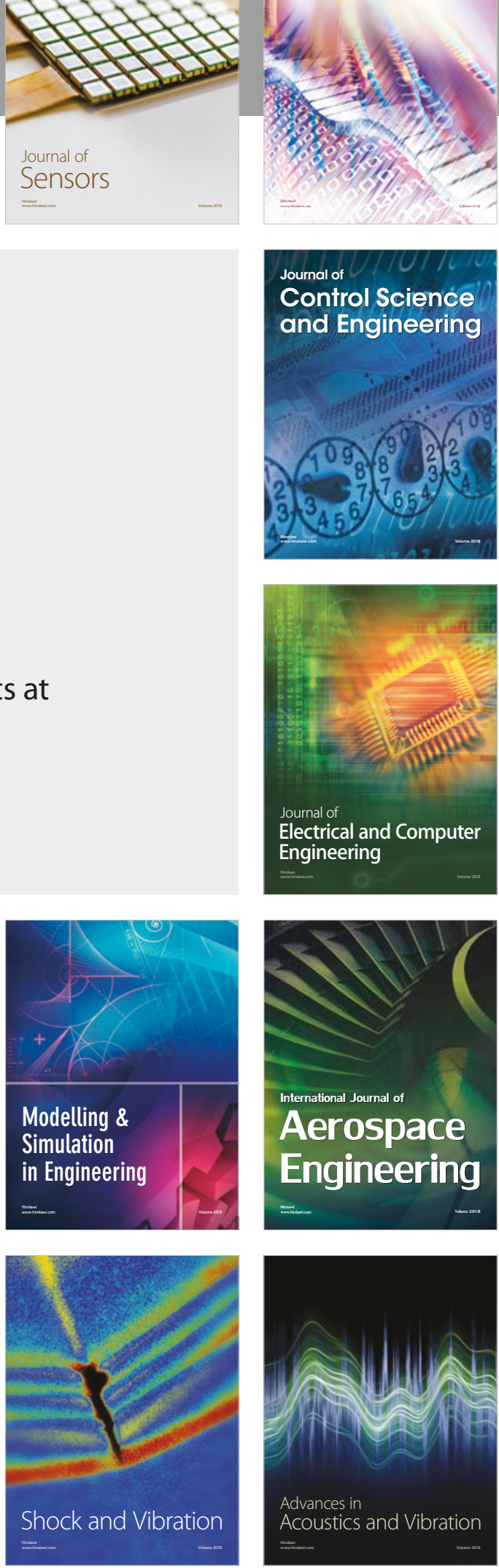